\title{
Palatal Cyst: An Unusual Case Report
}

\author{
Saurabh Agarwal, Mohan Jagade, Vandana Thorawade, Aseem Mishra, \\ Shreyas Joshi, Dnyaneshwar Ahire \\ Depatment of ENT \& Head \& Neck Surgery, Grant Medical College, Mumbai, India \\ Email: dr.saurabhagarwal@yahoo.com
}

Received October 25, 2012; received November 26, 2012; accepted December 5, 2012

\begin{abstract}
Cyst is a fluid accumulated in a cavity lined by epithelium. Cyst over the hard palate is very infrequent. Cyst is commonly seen along nasoalveolar duct or midline palatal cyst which are congenital. Only few cases of palatine cysts have been reported in literature. We present here a case of 21 years old male with a cystic lesion over the hard palate since 2 years.
\end{abstract}

Keywords: Palatal Cyst; Hard Palate; Nasopalatine Duct

\section{Introduction}

Palatine cysts are rare, non-odontogenic fissural cysts of the hard palate. These cysts occur in the midline of the hard palate, behind the incisive canal. These cysts are usually asymptomatic; however they can result in swelling, pain and discharge. The radiological imaging can reveal a round, oval or heart shaped well demarcated image which can be confounding with inflammatory lesions. Being defined to have collection of fluid within a cavity lined by epithelium, a variety of cysts is described. NPDCs are the most common nonodontogenic cysts of the mouth, representing upto $1 \%$ of all maxillary cysts [1]. These lesions are almost three times frequent in males than in females [2]. The maximum prevalence is between 40 and 60 years of age. Some are developmental and some are congenital in origin. Some varieties of cysts are known as their own entity because of their position. Only few case reports have documented these cysts. Knowledge of their existence is important and should not be confused with malignant tumors.

\section{Case Report}

A 21 years old male presents with a swelling over the hard palate since 2 years and was associated with pain over the swelling (Figure 1). On examination there was a small lesion of firm consistency of $1 \times 2 \mathrm{~cm}$ in size and oval in shape over the right paramedian position over the hard palate just behind the right upper lateral incisors. There was mild tenderness over the swelling. Rest of the oral and dental examination was normal.

$\mathrm{X}$-ray reveals a cystic lesion just behind the right upper lateral incisor (Figure 2). The patient was planned for cyst excision. The patient was taken for operation under local anesthesia. Cyst was enucleated in toto and primary closure was done. Post-operative period was uneventful. Histopathology examination reveals a fibrous wall lined by stratified squamous epithelium (Figure 3). Patient was followed up for a period of one year without any signs of recurrence.

\section{Discussion}

Cysts in the midline of the palate \& nasoalveolar or nasopalatine cysts are very uncommon $[2,4-8]$. The cysts in

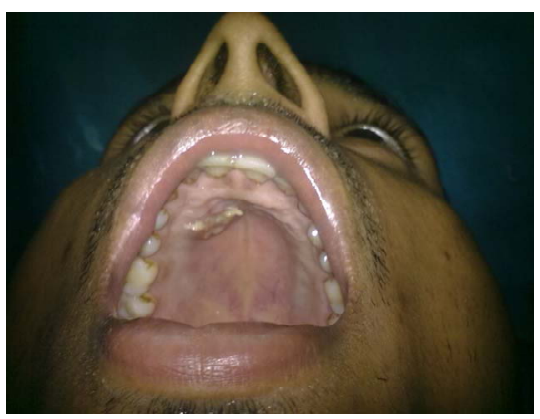

Figure 1. Cyst present over hard palate.

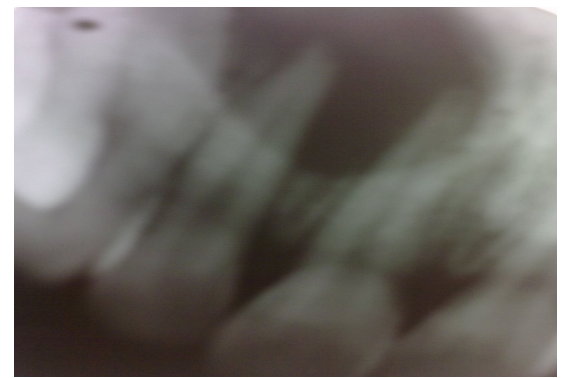

Figure 2. Occlusal radiograph showing palatal cyst. 


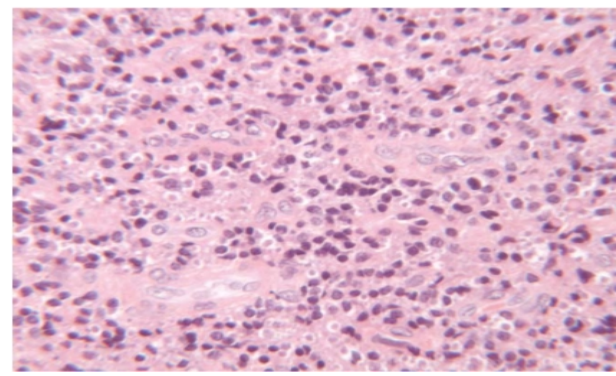

Figure 3. Photomicrograph of palatine cyst.

this region are usually an extension of cysts from adjacent regions, which involve or cross the midline. The cysts which arise from the midline and expand from there include median palatal cyst, nasopalatine or nasoalveolar cyst and nasopalatal duct cyst [9].

The nasopalatine duct cyst is a developmental cyst derived from proliferation of embryonic epithelial remnants of the nasopalatine duct. It may occur at any age but it is seen most often in fourth to sixth decades of life. The cause of nasopalatine duct cyst is essentially unknown. Trauma, infection, and mucous retention within associated salivary gland ducts have all been suggested as possible pathogenetic factors; however, most believe that spontaneous cystic degeneration of residual ductal epithelium is the most likely etiology. These are usually central or unilateral with no prevalence of side occurrence. Radiographically, some lesions may appear heartshaped, either because they become notched by the nasal septum during their expansion or because the nasal spine is superimposed on the radiolucent area.

A thorough differential diagnosis must be established in order to avoid unnecessary treatments such as endodontic procedures in vital permanent upper central incisors $[2,3]$. A correct tentative diagnosis should be based on positive vitality testing and negative percussion findings of the permanent upper central incisors, provided these teeth do not have pulp or periodontal problems [2]. In addition to panoramic X-rays, other complementary techniques are advised, such as periapical and occlusal $\mathrm{X}$-rays and computed tomography. The latter technique guarantees in establishing a tentative diagnosis, since it generates great detail of the structures (normally intact) adjacent to the lesion.

The differential diagnosis may include an enlarged nasopalatine duct (less than $6 \mathrm{~mm}$ in diameter), central giant cell granuloma, a radicular cyst associated to the upper central incisors, follicular cyst associated with mesiodens, primordial cyst, nasoalveolar cyst, osteitis with palatal fistulization, and bucconasal and/or buccosinusal communication [3].

Median alveolar or midline anterior cyst which is usually found in incisive foramen region is a controversial fissural cyst [1]. The fact that no epithelial remnant exist due to the fusion of embryonic processes rules out the possibility of such a cystic origin $[10,11]$.

However few cases of median palatine cysts have been reported which may accidentally be found to be present on routine radiographic examination. Approximately about 20 - 30 cases have been reported in last 40 years [6]. Most of them are asymptomatic, but when symptomatic they present with a swelling on the palate either in midline or adjacent to it, however lying posterior to the incisive papilla. One feature common to this cyst is presence of vital teeth adjacent to the lesion and residual or periapical cyst [4].

The histopathologic examination of the cystic lining revealed fibrous wall lined by thin stratified squamous epithelium and partly by pseudo stratified columnar epithelium. A few nerve bundles and blood vessels were seen in cyst. These histological features, in conjunction with the site of lesion, suggested palatine cyst, which is regarded as a rare entity $[1,6]$.

\section{Conclusion}

The present case is of particular clinical interest as Palatine cysts are rare and it is important that clinician should be aware of the features of this cyst as nearly $40 \%$ of the cases are totally asymptomatic and found only during routine clinical examination. Due to extent of the lesion, surgical enucleation was the choice of treatment. Our case demonstrated typical clinical, radiographical and histopathological features of palatine cyst.

\section{REFERENCES}

[1] N. Ely, E. C. Sheehy and F. McDonald, "Nasopalatine Duct Cyst: A Case Report,” International Journal of Paediatric Dentistry, Vol. 11, No. 2, 2001, pp. 135-137. doi:10.1046/j.1365-263x.2001.00248.x

[2] J. D. Gnanasekhar, S. V. Walvekar, A. M. Al-Kandari and Y. Al-Duwairi, "Misdiagnosis and Mismanagement of a Nasopalatine Duct Cyst and Its Corrective Therapy: A Case Report," Oral Surgery, Oral Medicine, Oral Pathology, Oral Radiology and Endodontology, Vol. 80, No. 4, 1995, pp. 465-470. doi:10.1016/S1079-2104(05)80372-5

[3] H. D. Moss, J. W. Hellstein and J. D. Johnson, "Endodontic Considerations of the Nasopalatine Duct Region," Journal of Endodontics, Vol. 26, No. 2, 2000, pp. 107110. doi:10.1097/00004770-200002000-00012

[4] H. Kato, M. Kanematsu, Y. Kusunoki, T. Shibata, H. Murakami, K. Mizuta, Y. Ito and Y. Hirose, "Nasoalveolar Cyst: Imaging Findings in Three Cases,” Clinical Imaging, Vol. 31, No. 3, 2007, pp. 206-209. doi:10.1016/j.clinimag.2006.12.026

[5] R. Hegde and R. Shetty, "Nasopalatine Duct Cyst," Journal of Indian Society of Pedodontics and Preventive Dentistry, Vol. 24, No. 5, 2006, p. 31. 
[6] A. A. Sazgar, M. Sadeghi, A. K. Yazdi and L. Ojani, "Transnasal Endoscopic Marsupialization of Bilateral Nasoalveolar Cysts," International Journal of Oral and Maxillofacial Surgery, Vol. 38, No. 11, 2009, pp. 12101211. doi:10.1016/j.ijom.2009.06.012

[7] A. Erkan, C. Ylmazer, I. Ylmaz and F. Bolat, "Nasoalveolar Cysts: Review of 3 Cases,” ORL: Journal for OtoRhino-Laryngology, Vol. 67, No. 4, 2005, p. 196. doi:10.1159/000086664

[8] G. Courage, A. North and L. Hansen, "Median Palatine Cysts: Review of the Literature and Report of a Case," Oral surgery, Oral Medicine, Oral Pathology, Vol. 37,
No. 5, 1974, pp. 745-753. doi:10.1016/0030-4220(74)90140-6

[9] R. Vasconcelos, M. Aguiar, W. Castro, V. Araújo and R. Mesquita, "Retrospective Analysis of 31 Cases of Nasopalatine Duct Cyst,” Oral Diseases, Vol. 5, No. 4, 2008, pp. 325-328. doi:10.1111/j.1601-0825.1999.tb00098.x

[10] P. Scolozzi, A. Martinez, M. Richter and T. Lombardi, “A Nasopalatine Duct Cyst in a 7-Year-Old Child,” Pediatric Dentistry, Vol. 30, No. 6, 2008, pp. 530-534.

[11] A. Meyer, “A Unique Supernumerary Paranasal Sinus Directly above the Superior Incisors," Journal of Anatomy, Vol. 48, 1914, pp. 118-129. 